\title{
Sur les hypersurfaces minimales dans un espace elliptique à quatre et à cinq dimensions.
}

\author{
R. Rosca, L. Vanhecke et I. Verstratelen $(*)$ (Heverlee, Belgique) $(* *)$
}

\begin{abstract}
Résumé. - On étude des hypersurfaces minimales dans un espace elliptique à quatre et à cinq dimensions. L'introduction de certaines notions qui ont trait à des relations d'invariance entre champs de vecteurs et r-formes permet de traduire quelques propriétés de ces variétés liées à ces relations d'invariance.
\end{abstract}

\section{Introduction.}

De nombreux travaux [6] on été consacrés au cours des ces dernières années aux variétés minimales de l'espace euclidien $E^{n}$ à $n$ dimensions et ont donné à cette théorie un renouveau d'intérêt. L'introduction dans l'étude actuelle de certaines notions qui ont trait à des relations d'invariance entre champs de vecteurs et $r$-formes nous a permis de traduire quelques propriétés géométriques de ces variétés liées à ces relations d'invariance. C'est ainsi que la considération d'après $R$. RoscA [11] de la 1-forme distinguée $\omega$ sur une variété, nous a permis de donner pour toute hypersurface minimale la définition suivante: Toute hypersurface $V^{n}$ immergée dans un espace riemannien quelconque pour laquelle $\omega$ est une relation intégrale d'invariance pour le champ de courbure (qui a pour composantes les courbures principales de la variété) de $V^{n}$, est minimale.

En vertu du principe de la dualité, qui est fondamental dans l'espace elliptique, il est bien connu que dans $P_{b}^{*}$ la duale d'une surface minimale est aussi minimale. Cette propriété n'est plus vraie en général pour $n>2$, mais l'on démontre que pour toute $V^{3} \subset P_{e}^{4}$ minimale qui se reproduit par dualité, les couples d'hypersurfaces parallèles et rectangulaires de $V^{3}$ sont des hypersurfaces $W$.

Le cas d'une $V^{3}$ homogène, connexe et dont le nombre type est 2 , immergée dans une sphère $S^{4}$ a été étudié récemment par R. TaKaGI [18], qui démontre qu'une pareille variété est nécessairement minimale. Dans l'espace $P_{s}^{4}$ ces variétés ont la propriété que leur duale est une variété bidimensionnelle minimale et isométrique au plan hyperbolique de courbure -1 .

Dans la deuxième partie de cet ouvrage, on étudie les $V^{4} \subset P_{e}^{4}$ minimales qui sont, conformément à un théorème d'OTSSUKI [7], le produit riemannien local de deux

(*) Ce travail a été réalisé dans le cadre de l'accord culturel entre la Belgique et la Roumanie.

(**) Entrata in Redazione il 5 novembre 1971 . 
2-sphères de courbure 2. Dans un certain cas particulier on définit sur une pareille $V^{4} \subset P_{e}^{5}$ une 2-forme distinguée (dans le sens de R. Rosca [12]) qui est symplectique et pour laquelle le champ de courbure est hamiltonien.

\section{Section I}

1. - Soit $P_{a}^{4}$ un espace elliptique à quatre dimensions dont on suppose que la courbure a été réduite à l'unité par une homothétie préalable et soit $\pi: V^{3} \rightarrow P_{e}^{4}$ une immersion isométrique d'une $C^{\infty}$-hypersurface orientable.

$\boldsymbol{X}_{0}$ étant le point générique de $V^{\hat{\imath}}$, associons à $\boldsymbol{X}_{0}$ un repère orthonormé (ou pentaèdre rectangle) $S_{5} \equiv\left\{\boldsymbol{X}_{A}\right\}, \quad(A, B=0,1,2,3,4)$. L'espace tangent dual $T\left(\boldsymbol{X}_{0}\right)$ en $\boldsymbol{X}_{0}$ à $V^{3}$ ètant déterminé par $\boldsymbol{X}_{i}(i, j, k=1,2,3)$, soit $\omega^{i}\left(u^{j} \mid d u^{j}\right)$ la base duale de $T\left(\boldsymbol{X}_{0}\right)$. Dans ces conditions la variété $V^{3}$ est structurée par la connexion

$$
d \boldsymbol{X}_{A}=\omega_{A}^{B} \boldsymbol{X}_{B}
$$

où $\omega_{A}^{B}$ sont des 1-formes de connexion de l'immersion $\pi$.

Supposons, ce qui est toujours possible, que la seconde forme fondamentale

$$
\varphi=-\left\langle d \mathbf{X}_{0}, d \mathbf{X}_{4}\right\rangle=\omega_{i}^{4} \omega^{i}
$$

est diagonale. Dans ce cas, on peut écrire

$$
\varphi=\sum_{i} k_{i}\left(\omega^{i}\right)^{2}
$$

$k_{i}$ étant, comme on le sait, les trois courbures principales de la variété $V^{3}$.

Dénommons le champ $K\left(k_{i}\right) \in T\left(\boldsymbol{X}_{0}\right)$ et la 1-forme $\omega \in \Lambda^{1}\left(V^{3}\right)$, définie par

$$
\omega=\sum_{i} \omega^{i}
$$

respectivement le champ de courbure et la 1-forme distinguée [11] de la variété $V^{2} \subset P_{e^{*}}^{4}$ En supposant que $\omega$ est une relation intégrale d'invariance du champ $\boldsymbol{K}$ dans le sens de A. LICHNÉRowicz [5], on devra écrire

$$
\boldsymbol{K}\rfloor \omega=0
$$

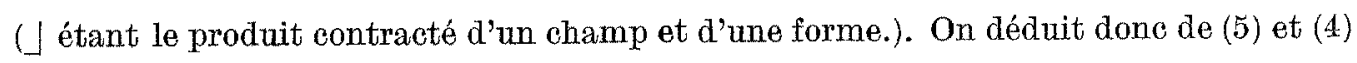

$$
\sum_{i} k_{i}=0,
$$


condition qui exprime le caractère minimal de la variété. Il est clair que cette formule reste valable dans le cas d'une hypersurface $V^{n} \subset P_{e}^{n+1}$ et l'on peut par conséquent formuler la proposition générale suivante:

Toute hypersurface orientable, pour laquelle la 1-forme distinguée est une relation intégrale d'invariance pour le champ de courbure, est minimate.

2. - La forme $\varphi$ étant diagonale, il est bien connu que $\omega^{i}=0$ est une sousvariété (dans le cas actuel une surface) de courbure de $V^{3}$. Conformément à la définition donnée dans [1], les sous-variétés de courbure sont totalement holonomes si ehacune des équations $\omega^{i}=0$ est complètement intégrable.

Le premier groupe des équations de structure associé à l'immersion $\pi$ étant

$$
d \wedge \omega^{i}=\omega^{j} \wedge \omega_{j}^{i}
$$

$\left(\omega_{j}^{i}=\gamma_{j k}^{i} \omega^{k} ; \gamma_{j k}^{i}=\right.$ coefficients de connexion), on trouve facilement que les trois conditions pour que les sous-variétés de courbure soient totalement holonomes sont

$$
\gamma_{j k}^{i}=0
$$

$(i, j, k$ est une permutation circulaire de $1,2,3)$.

Dans cette hypothèse, cherchons sous quelles conditions le champ $\boldsymbol{K}$ est un champ caractéristique de la forme distinguée $\omega$. Conformément à la définition d'un champ caractéristique (voir aussi [3]), on doit écrire en plus de (5):

$$
\boldsymbol{K}\rfloor(d \wedge \omega)=0 .
$$

En effectuant le calcul indiqué par (9) on trouve

$$
k_{i}=\varrho A_{i}, \quad \varrho \in \mathbb{D}\left(V^{3}\right)
$$

où

$$
A_{i}=\gamma_{j i}^{k}-\gamma_{k \hbar k}^{j} .
$$

$(i, j, k$ est de nouveau une permutation circulaire de 1, 2, 3.) En vertu de la relation (6) qui exprime que l'hypersurface est minimale, il résulte que

$$
\sum A_{i}=0 \Leftrightarrow \omega \wedge(d \wedge \omega)=0 .
$$

Ainsi: si le champ de courbure de l'hypersurface est un champ caractéristique de la forme distinguée, l'équation $\omega=0$ est nécessairement complètement intégrable et ceci prouve l'existence des surfaces définies par $\omega=0$. 
Signalons que, si $\boldsymbol{K}$ n'est pas earactéristique (ou autrement dit si $\omega$ est une forme de contact), conformément au théorème de REes [10], le champ $\boldsymbol{D}$ de composantes $\varrho A_{i}$, où $1 / \varrho=\sum_{i} A_{i}$, est te champ dynamique correspondant à la forme $\omega=0$.

3. - Il est bien connu que dans l'espace elliptique tridimensionnel la surface duale d'une surface minimale est aussi minimale. Il est facile de voir que, quand il s'agit d'hypersurfaces minimales, cette propriété n'est plus vraie en général. Dans le cas que nous étudions et en nous rapportant à la connexion (1), on voit que la variété tangentielle duale de $\left(\boldsymbol{X}_{4}\right)$ en $\boldsymbol{X}_{4}$ est la même que celle de $\left(\boldsymbol{X}_{0}\right)$ en $\boldsymbol{X}_{0}$. Du double fait que $\varphi$ est la seconde forme fondamentale commune de $\left(\boldsymbol{X}_{0}\right)$ et $\left(\boldsymbol{X}_{4}\right)$ et qu'elle est diagonale, on déduit que les courbures de $1^{1}$ hypersurface $* V^{3} \equiv\left(\boldsymbol{X}_{4}\right)$ sont $1 / k_{i}$. Par conséquent celle-ci est minimale si et seulement si la fonction symmétrique élémentaire du deuxième degré des courbures principales, à savoir

$$
\sum_{i, j(i \neq j)} k_{i} k_{j}
$$

est nulle.

Considérons dans ce cas l'hypersurface parallèle $\bar{V}^{3}$ de $V^{3}$, dont le point générique $\overline{\boldsymbol{X}}$ peut s'écrire

$$
\overline{\boldsymbol{X}}=\boldsymbol{X}_{0} \cos e+\boldsymbol{X}_{\mathbf{t}} \sin c \quad(c=\text { const })
$$

et dont la duale $* \bar{V}^{3}$ est manifestement décrite par

$$
* \overline{\boldsymbol{X}}=\boldsymbol{X}_{0} \sin c-\boldsymbol{X}_{4} \cos c .
$$

Un calcul facile donne pour les courbures principales $k_{i}$ de $(\overline{\boldsymbol{X}})$ :

$$
\vec{k}_{i}=\frac{\sin \theta+k_{i} \cos \theta}{\cos c-k_{i} \sin \theta}
$$

De (16) on déduit pour la courbure retative généralisée $\bar{K}$ de $\bar{V}^{3}$ (la fonction symmétrique élémentaire du troisième dégré des courbures principales) l'expression

$$
\bar{K}=\frac{\sin ^{3} e+K \cos ^{3} \theta}{\cos ^{3} \theta-K \sin ^{3} \theta}
$$

où $K$ est la courbure relative de $V^{3}$. De même on trouve que la courbure moyenne $\bar{H}$ de $\bar{V}^{3}$ est donnée par

$$
\bar{H}=\frac{\sin \theta \cos ^{2} c+K \cos \theta \sin ^{2} c}{\cos ^{3} c-K \sin ^{3} c} .
$$


L'élimination de $K$ donne aussitôt

$$
\bar{H}=\frac{\bar{K} \sin ^{2} c \cos ^{2} e+\sin c \cos c \cos 2 e}{1-3 \cos ^{2} c \sin ^{2} c}
$$

d'où le

THÉoRట̀ME. - Si l'hypersurface duale d'une hypersurface minimale $V^{3} \subset P_{e}^{4}$ est aussi minimale, toutes les hypersurfaces parallètes sont des hypersurfaces $W$.

Remarquons que les cas où $V^{3}$ est de courbure relative -1 (et puisqu'elle est en plus par hypothèse minimale), on peut la considérer comme une généralisation d'une surface de Clifford. Or, de (18) on déduit aussitôt que les hypersurfaces parallèles pour $c= \pm \pi / 4$ sont aussi minimales et de (17) on constate que $\bar{K}=0$. D'où le

Tmóorìme. - Si $V_{m}^{\mathbf{s}}$ est une hypersurface de Clifford, de même que sa duale, les deux hypersurfaces paralletes et rectangulaires pour $e= \pm \pi / 4$ sont minimales et a courbure relative nulle.

4. - Supposons maintenant que l'immersion $\pi$ se rapporte à une variété $V_{h}^{3}$ homogène, connexe et dont le nombre type [18] est égal à 2 . Il est aisé de voir que les résultats établis per TAKAGI [18] sont valables aussi pour une pareille variété dans un espace elliptique. Une variété $V_{h}^{3} \subset P_{e}^{4}$ sera dono définie par

$$
\begin{gathered}
\omega_{3}^{4}=0, \\
\omega_{1}^{3}=b \omega^{2}, \quad \omega_{2}^{3}=c \omega^{1}, \quad(b \text { et } c=\text { const })
\end{gathered}
$$

et

$$
\begin{gathered}
\omega_{1}^{4}=\gamma_{11} \omega^{1}+\gamma \omega^{2}, \quad \omega_{2}^{4}=\gamma \omega^{1}+\gamma_{22} \omega^{2}, \\
\gamma_{11} \gamma_{22}-\gamma^{2}=\varkappa, \quad(x=\text { const }) .
\end{gathered}
$$

Remarques.

a) Dans le cas actuel on suppose que $\varphi$ n'est plus diagonale $(\gamma \neq 0)$.

b) Dans le cas de l'espace elliptique on peut définir une hypersurface dont le nombre type est égal à 2 , comme une hypersurface dont la duale est une variété bidimensionnelle.

A l'aide de (7) et du second groupe d'équations de structure

$$
d \wedge \omega_{B}^{A}=\omega_{B}^{c} \wedge \omega_{C}^{A},
$$


on trouve par différentiation extérieure de $\left(20^{\prime \prime}\right)$

$$
b+c=0, \quad 1+b c=0
$$

et ceci est en accord avec le résultat de $R$. TAKagI [18] $\left(\omega_{1}^{2} \neq 0\right)$. Compte tenu de [24], la différentiation extérieure de $\left(20^{\prime}\right)$ donne

$$
\gamma_{11}+\gamma_{22}=0
$$

Ainsi, comme dans [18], une hypersurface homogène $V_{h}^{3} \subset P_{e}^{4}$ est minimale. Mais en vertu de la remarque $b$ ), les deux formes fondamentales de la surface $* V_{m}^{2}$, décrite par $\boldsymbol{X}_{4}$, sont

$$
{ }^{*} \varphi_{0}=-\left\langle d \boldsymbol{X}_{4}, d \boldsymbol{X}_{0}\right\rangle, \quad * \varphi_{3}=-\left\langle d \boldsymbol{X}_{4}, d \boldsymbol{X}_{3}\right\rangle
$$

En notant par

$$
* \omega^{1}=-\omega_{1}^{4}, \quad * \omega^{2}=-\omega_{2}^{4}
$$

la base duale de $* V^{2}$, les formes (25) s'écrivent développées:

$$
\begin{aligned}
& { }^{*} \varphi_{0}=\frac{1}{\varkappa}\left\{\gamma_{22}\left({ }^{*} \omega^{1}\right)^{2}+\gamma_{11}\left({ }^{*} \omega^{2}\right)^{2}-2 \gamma^{*} \omega^{1 *} \omega^{2}\right\} \\
& { }^{*} \varphi_{3}=\frac{b}{\varkappa}\left\{\gamma\left({ }^{*} \omega^{1}\right)^{2}-\gamma\left({ }^{*} \omega^{2}\right)^{2}-2 \gamma_{11}{ }^{*} \omega^{1 *} \omega^{2}\right\}
\end{aligned}
$$

La simple lecture de (27) montre que les traces de ces formes sont nulles, d'où il résulte que $* V^{2}$ est aussi minimale. De plus, en étendant à l'espace $P_{\theta}^{n}$ la notion de courbures d'ôtsuki d'une variété $V^{2}$ immergée dans un espace euclidien [8], les courbures d'ôtsuki de $* V^{2}$ sont définies par

$$
\begin{aligned}
& \omega_{1}^{0} \wedge_{2}^{0} \omega=\frac{1}{x} * \omega^{1} \wedge^{*} \omega^{2}, \\
& \omega_{1}^{3} \wedge \omega_{2}^{\mathbf{3}}=-\frac{e b}{x} * \omega^{1} \wedge \omega^{* 2} .
\end{aligned}
$$

Ces relation et (24) montrent aussitôt que les deux courbures d'ত̄tsuki sont égales à $1 / x$. On a done le

THÉоR亡̀ME. - La surface duale d'une hypersurface tridimensionnelle, homogène, connexe et de nombre type 2 , immergée dans un espace elliptique $P_{e}^{4}$ est, te même que $V_{h}^{3}$, minimale et à courbure relative constante. 
Etablissons maintenant le théorème d'existence relatif à la variété $V_{n}^{3}$ la plus générale. On pent poser en vertu de (22):

$$
\gamma_{11}=R \cos \theta, \quad \gamma=R \sin \theta, \quad R^{2}=-\varkappa
$$

dans ce cas les équations (21) s'écrivent

$$
\omega_{1}^{4}=R\left(\cos \theta \omega^{1}+\sin \theta \omega^{2}\right), \quad \omega_{2}^{4}=R\left(\sin \theta \omega^{1}-\cos \theta \omega^{2}\right) .
$$

En posant

$$
\omega_{1}^{2}=p \omega^{1}+q \omega^{2}+r \omega^{3},
$$

la différentiation extérieure de ces équations donne

$$
\begin{aligned}
& d \theta \wedge \omega^{2}+2 p \omega^{1} \wedge \omega^{2}+(b-2 r) \omega^{1} \wedge \omega^{3}=0 \\
& d \theta \wedge \omega^{1}-2 q \omega^{1} \wedge \omega^{2}-(b-2 r) \omega^{3} \wedge \omega^{1}=0
\end{aligned}
$$

d'où l'on déduit

$$
d \theta=-2 p \omega^{1}-2 q \omega^{2}+(b-2 r) \omega^{3} .
$$

De (34) on obtient

$$
\begin{aligned}
d p \wedge \omega^{1}+d q \wedge \omega^{2}+d r \wedge \omega^{3}+\left(p^{2}+q^{2}-b^{2}+2 b r\right) \omega^{1} \wedge \omega^{2}= & \\
& +p(b-r) \omega^{2} \wedge \omega^{3}-q(b-r) \omega^{1} \wedge \omega^{3}=0 .
\end{aligned}
$$

D'autre part la différentiation extérieure de (32) donne

$$
\begin{aligned}
-x \omega^{1} \wedge \omega^{2}=d p \wedge \omega^{1} & +d q \wedge \omega^{2}+d r \wedge \omega^{3}+ \\
& +\left(p^{2}+q^{2}+2 b r\right) \omega^{2} \wedge \omega^{2}+p(b-r) \omega^{2} \wedge \omega^{3}-q(b-r) \omega^{1} \wedge \omega^{2}
\end{aligned}
$$

et par comparaison avec (35) on obtient aussitôt

$$
R^{2}=b^{2}, \quad\left(b^{2}=1\right)
$$

Cette relation montre que dans l'espace $P_{e}^{4} \varkappa$ doit nécessairement être égal $\grave{a}-1$ et par conséquent

Toute $V_{\hbar}^{3}$ connexe et de nombre type 2 a pour variété duale une variété bidimensionnelle isométrique au plan hyperbolique de courbure -1 . 
Remarque. - La différentiation extérieure de $\omega_{1}^{2}$ donne

$$
d \wedge \omega_{1}^{2}=-(2+x) \omega^{1} \wedge \omega^{2}
$$

On pent done dire que la courbure intrinsèque de la sous-variété définie par $\omega_{0}^{3}=0$ est constante. Dans ce cas on a une sous-variété bidimensionnelle isométrique au plan elliptique.

La différentiation extérieure de (35) (qui est l'unique équation du deuxième degré) donne l'équation du troisième degré:

$$
p d p \wedge \omega^{1} \wedge \omega^{2}+q d q \wedge \omega^{1} \wedge \omega^{2}-p d r \wedge \omega^{2} \wedge \omega^{3}+q d r \wedge \omega^{1} \wedge \omega^{3}=0 .
$$

Le système extérieur $\Sigma$, défini par $\left(20^{\prime}\right),\left(20^{\prime \prime}\right),(31),(32),(34),(35)$ et $(38)$, pour nombres caractéristiques

$$
r=10, \quad s_{0}=7, \quad s_{1}=1, \quad s_{2}=1, \quad s_{3}=1 .
$$

Conformément au test de Cartan, le système $\Sigma$ en question est en involution et on arrive au

THéonème. - Toute $V_{h}^{3} \subset P_{c}^{4}$ connexe et do nombre type 2 est déterminée par un système en involution avec l'arbitraire d'une fonetion de trois arguments.

Si l'on écrit les équations de Laplace non-holonomes relatives au réseau isotrope ayant pour support la surface $* V_{m}^{2}$, on trouve le résultat prévisible [13] que ce réseau est à invariants égaux et constants $\left(=\frac{1}{2}\right)$. Mais ce qu'il y a de plus important se rapporte aus transformées de Laplace non-holonomes du réseau isotrope. En notant par $\boldsymbol{Y}_{1}, \boldsymbol{Y}_{2}$ ces transformées, un calcul facile donne:

$$
\boldsymbol{Y}_{1}=\frac{1}{2}\left(\boldsymbol{X}_{1}-i \boldsymbol{X}_{2}\right), \quad \boldsymbol{Y}_{2}=\frac{1}{2}\left(\boldsymbol{X}_{1}+i \boldsymbol{X}_{2}\right)
$$

Il est visible que ces points sont des points isotropes, e'est-à-dire qu'ils sont situés sur l'absolu $Q$ qui définit la métrique de $P_{e}^{4}$. En différentiant $\boldsymbol{Y}_{1}$ et $\boldsymbol{Y}_{2}$, on obtient à l'aide de la connexion (1):

$$
\left\{\begin{array}{l}
d \boldsymbol{Y}=2 i \omega_{1}^{2} \boldsymbol{Y}_{1}-\bar{\alpha}\left(\boldsymbol{X}_{0}-i \boldsymbol{X}_{3}\right)+\left(\gamma_{11}-i \gamma\right) \alpha \boldsymbol{X}_{4}, \\
d \boldsymbol{Y}_{2}=-2 i \omega_{1}^{2} \boldsymbol{Y}_{2}-\alpha\left(\boldsymbol{X}_{0}+i \boldsymbol{X}_{3}\right)+\left(\gamma_{11}+i \gamma\right) \alpha \boldsymbol{X}_{4},
\end{array}\right.
$$

où

$$
\alpha=\omega^{1}+i \omega^{2}, \quad \bar{\alpha}=\omega^{1}-i \omega^{2} .
$$

En rappelant que $\omega_{1}^{2}$ est une forme linéaire de $\omega^{1}, \omega^{2}, \omega^{3}$ et en remarquant que 
$\boldsymbol{X}_{0}+i \boldsymbol{X}_{3}\left(\boldsymbol{X}_{0}-i \boldsymbol{X}_{3}\right), \boldsymbol{Y}_{1}, \boldsymbol{Y}_{2}$ sont des points isotropes et rectangulaires, on déduit que $\boldsymbol{Y}_{1}, \boldsymbol{Y}_{2}$ sont les points génériques de deux hypersurfaces isotropes de défaut 2 , d'où le

THÉoRł̀ne, - Les transformées de Laplace non-holonomes du réseau isotrope ayant comme support la surface duale d'une variété $V_{h}^{3}$ connexe et de nombre type 2 sont des hypersurfaces isotropes de défaut 2 tracées sur l'absolu $Q$.

Soit maintenant le point $Z \in T\left(X_{4}\right)$

$$
\boldsymbol{Z}=\boldsymbol{X}_{1} \cos \varphi+\boldsymbol{X}_{\mathbf{2}} \sin \varphi
$$

Ce point définit une autotransformation infinitésimale équivalente $\left[\boldsymbol{X}_{\mathbf{4}} \rightarrow \boldsymbol{X}_{\mathbf{4}}+\varepsilon \boldsymbol{Z}\right.$, $\varepsilon$ constante infinitésimale] de $\boldsymbol{X}_{4}$ \{voir aussi [19], [14]\}, sous la condition nécessaire et suffisante

$$
\Delta\left(\boldsymbol{X}_{4}, \boldsymbol{Z}\right)=\mathbf{0}
$$

$\triangle$ étant le paramètre différentiel mixte de Beltrami relatif aux pfaffiens invariants $\omega_{1}^{4}, \omega_{2}^{4} ;\left\{\boldsymbol{X}_{4}, \boldsymbol{Z}\right\}$ est la directrice de l'autotransformation. En introduisant $\boldsymbol{Z}$ dans (43), on trouve que la condition nécessaire et suffisante pour que $\boldsymbol{Z}$ définisse une transformation infinitésimale équivalente est

$$
\left(\omega_{1}^{2}+d \varphi\right) \wedge\left(\omega_{4}^{1} \cos \varphi+\omega_{4}^{2} \sin \varphi\right)=0
$$

De là il résùlte

$$
\omega_{1}^{2}+d \varphi=f\left\{\omega^{2} \cos (\theta-\varphi)+\omega^{2} \sin (\theta-\varphi)\right\}, \quad f \in \mathbb{D}\left(V^{3}\right),
$$

et par différentiation extérieure, compre tenu de (34), il vient

$$
\left(1-f^{2}\right) \omega^{1} \wedge \omega^{2}+d f \wedge\left\{\omega^{1} \cos (\theta-\varphi)+\omega^{2} \sin (\theta-\varphi)\right\}=0 .
$$

On déduit de $\left(44^{\prime \prime}\right)$ que pour $f= \pm 1$ le système $\Sigma$ continue à être en involution avec le même arbitraire, car par le problème posè, il s'introduit une nouvelle forme $d \varphi$ et une nouvelle équation linéaire (44'). Par conséquent on peut dire que dans la cas où $f= \pm 1$, l'angle $\varphi$, défini par $\left(44^{\prime}\right)$, définit bien une autotransformation infinitésimale équivalente de $\left(\boldsymbol{X}_{4}\right)$.

5. - On peut lier les développements du paragraphe précédent à la notion de variété pseudo-isotrope introduite par $R$. Rosca dans [15], [16], [20] et [21]. Les formes métriques de différentes variétés immergées dans $P_{e}^{n}$ étant des formes définies positives, il est clair que les variétés isotropes ou pseudo-isotropes d'un espace $P_{\bullet}^{n}$

24 - Annali ai Matematica 
sont complexes. Il est bon de noter aussi que dans l'espace elliptique où le principe de la dualité joue un rôle important, toute variété pseudo-isotrope peut se définir conformément à la définition donnée dans [15] et [16] comme une hypersurface dont la duale est isotrope.

Dans ces conditions, supposons que l'immersion $\pi$ se rapporte à un $V^{3}$ de nombre type 2. Dire que $V^{3}$ est pseudo-isotrope reviènt à écrire:

$$
\left\langle d \boldsymbol{X}_{4}, d \boldsymbol{X}_{4}\right\rangle=0
$$

Puisque par hypothèse le nombre type est 2 , on a

$$
\omega_{3}^{2}=0
$$

et l'on déduit de (45):

$$
\omega_{1}^{4}= \pm i \omega_{2}^{4}
$$

On peut sans restriction supposer que $\omega_{1}^{4}=i \omega_{2}^{4}$. Dans ce cas et compte tenu de $(30)$, il vient

$$
\gamma_{11}=i \gamma, \quad \gamma_{22}=-i \gamma,
$$

et de là on déduit que toutes les courbures principales sont nulles et l'on arrive par conséquent au résultat prévisible [22] que la variété $V^{3}$ singulière en question est un hyperplan.

\section{Section II.}

1. - Supposons maintenant que l'immersion $\pi$ se rapporte à une hypersurface 4-dimensionnelle $V_{m}^{4}$ de $P_{e}^{5}$, possédant conformément à la terminologie d'ŌTsUKI [8] deux courbures principales. Notons une pareille variété par $\nabla_{m}^{4}(2,2)$ et posons

$$
k_{1}=k_{2}=\lambda, \quad k_{3}=k_{4}=\mu, \quad \lambda+\mu=0 .
$$

Les considérations de I.1 montrent qussitôt que la variété duale d'une $V_{m}^{4}(2,2)$ est aussi une $V_{m}^{4}(2,2)$, qui sera notée $* V_{m}^{4}(2,2)$.

Conformément au théorème d'ötsuki [7], une pareille hypersurface est le produit riemannien local de deux 2 -spheres de courbure 2 et l'on peut écrire

$$
\nabla_{m}^{4}(2,2) \simeq S^{2}\left(\sqrt{\frac{1}{2}}\right) \times S^{2}\left(\sqrt{\frac{1}{2}}\right) .
$$


2. - Soient $V^{4}$ et $* V^{4}$ les deux hypersurfaces rectangulaires et parallèles à $V_{m}^{4}(2,2)$ dont les points génériques peuvent s'écrire respectivement

$$
\overline{\boldsymbol{X}}=\boldsymbol{X}_{0} \cos c+\boldsymbol{X}_{5} \sin c, \quad * \bar{X}=\boldsymbol{X} \sin c-\boldsymbol{X}_{5} \cos c, \quad(c=\text { const }) .
$$

Par un calcul analogue à celui du T.3 on trouve

$$
\bar{k}_{i}=\frac{\sin c+k_{i} \cos c}{\cos c-k_{i} \sin c} .
$$

De là on déduit pour la courbure relative $\bar{K}$ et la courbure moyenne $\vec{H}$ de $\bar{V}^{a}$ les expressions

$$
\begin{gathered}
\bar{K}=\frac{\sin ^{4} e+K \cos ^{4} c+S_{2} \cos ^{2} c \sin ^{2} c}{\cos ^{4} e+K \sin ^{4} c+S_{2} \cos ^{2} c \sin ^{2} c} \\
\bar{H}=\frac{\sin c \cos ^{3} c-K \cos c \sin ^{3} c-\frac{1}{2} S_{2}\left(\cos ^{3} c \sin e-\cos c \sin ^{3} c\right)}{\cos ^{4} c+K \sin ^{4} c+S_{2} \sin ^{2} c \cos ^{2} c}
\end{gathered}
$$

où

$$
B_{2}=\sum k_{i} k_{j}
$$

Si $\sigma$ est la norme de la seconde forme fondamentale (ou le carré de la longueur la seconde forme fondamentale) de l'immersion $\pi$ [4], celle-ci est comme on sait

$$
\sigma=\sum_{i, j}\left(\gamma_{i j}^{5}\right)^{2}
$$

Supposons que le nombre $\sigma$ soit égal au nombre de dimension de l'hypersurface en question. On devra donc écrire

$$
\sum_{i} k_{i}^{2}=4
$$

Cette relation caractérise une hypersurface minimale du type de $K$. KenMotsu [4]. Conformément au théorème établi dans [4], $\nabla_{m}^{4}(2,2)$ est dans ce cas une sous-variété ouverte d'une des variétés $V_{r, n-r}$ et l'on trouve

$$
\lambda^{2}=1
$$

ou

$$
\lambda=1, \quad \mu=-1
$$


Ainsi:

Pour une hypersurface $V_{m}^{4}(2,2)$ qui est aussi du type de Kenmotsu, toutes les hypersurfaces parallèles sont de courbure mouenne constante $(\bar{H}=\operatorname{tg} 20)$ et de plus la courbure relative est égale $\dot{a}+1$.

3. - Faisons comme dans I.2 l'hypothèse que les sous-variétés de courbure de $V_{m}^{4}(2,2)$ sont totalement holonomes. On trouve par un calcul analogue que les formes de connexion tangentielles sont exprimées par

$$
\begin{aligned}
& \omega_{1}^{2}=\gamma_{11}^{2} \omega \omega^{3}+\gamma_{12}^{2} \omega^{2}, \\
& \omega_{1}^{3}=\gamma_{11}^{3} \omega^{x}+\gamma_{13}^{3} \omega^{3}, \\
& \omega_{2}^{3}=\gamma_{22}^{3} \omega^{2}+\gamma_{23}^{3} \omega^{3}, \\
& \omega_{1}^{4}=\gamma_{11}^{4} \omega \omega^{1}+\gamma_{14}^{4} \omega^{4}, \\
& \omega_{2}^{4}=\gamma_{22}^{4} \omega^{2}+\gamma_{24}^{4} \omega^{4}, \\
& \omega_{3}^{4}=\gamma_{33}^{4} \omega \omega^{3}+\gamma_{34}^{4} \omega \omega^{4},
\end{aligned}
$$

Dans ces conditions les différentielles extérieures des formes duales non-nulles s'ecrivent

$$
d \wedge \omega^{i}=\omega^{i} \wedge \sum_{j} \gamma_{i i}^{j} \omega^{j}, \quad(i=1,2,3,4)
$$

D'autre part, conformément à la définition donnée dans [13], on peut définir les quasi-asymptotiques d'une $V^{4} C P_{s}^{5}$ (roir aussi [17]) par l'équation

$$
\left|\boldsymbol{X}_{0} \boldsymbol{X}_{1} \boldsymbol{X}_{2} \boldsymbol{X}_{3} \boldsymbol{X}_{4} d^{3} \boldsymbol{X}_{0}\right|=0
$$

L'équation

$$
\left|\boldsymbol{X}_{5} \boldsymbol{X}_{1} \boldsymbol{X}_{2} \boldsymbol{X}_{3} \boldsymbol{X}_{4} d^{3} \boldsymbol{X}_{5}\right|=0
$$

est celle des quasi-asymptotiques de l'hypersurface duale. En faisant usage de la connexion $\mathrm{I}(1)$ et en négligeant les différentielles algébriques $d \omega_{B}^{A}$, on trouve après calculs, dans l'hypothèse que $X_{0}$ est le point générique d'une $V_{m}^{4}(2,2)$, que l'équation (13) s'écrit

$$
\sum_{i, j, k} \gamma_{i j}^{k} \omega^{i} \omega^{j} \omega^{k}=0
$$


R. Rosca - L. VANHECKE - L. VRRstraelex : Sur les hypersurfaces, ete. 373

Dans (15) les indices sont pris d'une telle manière qu'ils n'appartiennent jamais tous au même groupe $(1,2)$ ou $(3,4)$. Il est facile de voir que cette équation correspond aussi aux quasi-asymptotiques de la variété $* V_{m}^{4}(2,2)$.

Etudions maintenant un cas particulier qui donne lieu à des développements qui se lient à des considérations sur la théorie des champs sur les variétés symplectiques. Dans ce but, vu le rôle distingué que jouent les groupes d'indices $(1,2)$ et $(3,4)$ qui correspondent anx deux courbures, faisons l'hypothèse supplémentaire que les différentielles extérieures des formes duales qui correspondent à chaque groupe d'indices sont conformes pour ce groupe. Cela entraîne conformément à (12) la nullité des $\gamma_{i i}^{i}$. On a done

$$
\gamma_{i t}^{i}=0
$$

avee la même règle pour les indices que dans la formule (15). Dans ces conditions l'équation (15) des quasi-asymptotiques est identiquement nulle et l'on arrive au

THÉoRÈnr. - Si les différentielles des formes duales d'une variété $V_{m}^{4}(2,2)$ qui correspondent à chaque groupe d'indices sont conformes et si le système de sous-variétés de courbure est totatement holonome, toute courbe de $V_{m}^{4}(2,2)$ et toute courbe de $* V_{m}^{4}(2,2)$ est une quasi-asymptotique.

Soit maintenant la 2 -forme $\Omega \in \Lambda^{2}\left(V_{m}^{4}(2,2)\right)$ définie par [12]

$$
\Omega=\omega^{1} \wedge \omega^{2}+\omega^{3} \wedge \omega^{4} .
$$

Il est visible que $(\bigwedge \Omega)^{2}$ est une forme volume. D'autre part, en vertu des formules précédentes, on trouve

$$
d \wedge \Omega=0
$$

Cette propriété, ajoutée à la précédente, exprime comme on le sait, que $\Omega$ est une forme symplectique sur $V_{m}^{4}(2,2)$. Notons par $T\left(\boldsymbol{X}_{0}\right)=\left\{X_{i} ; i=1,2,3,4\right\}$ l'espace tangent dual en $X_{0} \in V_{m}^{4}(2,2)$. On peut conformément à la définition générale, définir un champ hamiltonien $\boldsymbol{h}\left(h_{i}\right) \in T\left(\boldsymbol{X}_{0}\right)$ par la condition

$$
d(\boldsymbol{h}\rfloor \Omega)=0
$$

Dans le cas particulier où le champ $\boldsymbol{h}$ est le champ de courbure, l'équation (19) devient

$$
d \lambda \wedge\left(\omega^{2}-\omega^{1}-\omega^{4}+\omega^{3}\right)+\left(\gamma_{12}^{2}-\gamma_{11}^{2}\right) \omega^{1} \wedge \omega^{2}-\left(\gamma_{34}^{4}-\gamma_{33}^{4}\right) \omega^{3} \wedge \omega^{4}=0
$$

D'autre part, la différentiation extérieure de deux des équations $\omega_{i}^{\mathbf{5}}$ donne aussitôt 
$\lambda=$ const. L'équation $(20)$ se réduit dans ce cas à

$$
\left(\gamma_{12}^{2}-\gamma_{11}^{2}\right) \omega^{1} \wedge \omega^{2}-\left(\gamma_{34}^{4}-\gamma_{33}^{4}\right) \omega^{3} \wedge \omega^{4}=0
$$

Cette équation du deuxième degré entraîne les conditions sous lesquelles les formes $\omega^{1}$, $\omega^{2}$ resp. $\omega^{3}, \omega^{4}$ sont homologues, d'où le

THÉonème. - La condition nécessaire et suffisante pour que le champ de courbure soit un ohamp hamiltonien sur la variété symplectique $\left(V_{m}^{4}(2,2), \Omega\right)$ est que les formes $\omega^{1}, \omega^{2}$ resp. $\omega^{3}, \omega^{4}$ soient homologues.

4. - Notons par $C\left(c_{i}\right)$ et $\bar{C}\left(c_{i}\right)$ deux champs orthogonaux et unitaires qui appartiennent à $T\left(\boldsymbol{X}_{0}\right)$ et notons par $\theta(\boldsymbol{C})$ l'operateur $d^{\prime} \bar{\partial} t s u k i[9]$ qui peut être défini pour un espace elliptique par

$$
\theta(\boldsymbol{C})=\sum_{i, c} \gamma_{i j}^{5} c_{j} \boldsymbol{X}_{i}
$$

En vertu des hypothèses faites, la courbure de sectionnement $K(\pi)$ en $\boldsymbol{X}_{0} \in V_{m}^{4}$ pour l'élément de plan tangent $\pi$, défini par deux champs unitaires orthogonaux $\boldsymbol{C}$ et $\overline{\boldsymbol{C}}$, a pour expression

$$
K(\pi)=\frac{P}{G}
$$

où

$$
\begin{aligned}
& P=\langle\theta(\boldsymbol{C}), \boldsymbol{C}\rangle\langle\theta(\overline{\boldsymbol{C}}), \overline{\boldsymbol{C}}\rangle-\langle\theta(\boldsymbol{C}), \overline{\boldsymbol{C}}\rangle^{2}, \\
& G=\|\boldsymbol{C}\|^{2}\|\overline{\boldsymbol{C}}\|^{2}\|-\|\langle\boldsymbol{C}, \overline{\boldsymbol{C}}\rangle \|^{2} .
\end{aligned}
$$

Avec les hypothèses faites plus haut et en remplaçant dans (23) on trouve

$$
K(\pi)=2 \lambda^{2}\left(A_{12}+A_{34}\right)-\lambda^{2}
$$

où

$$
A_{12}=\left(c_{1} \bar{c}_{2}-c_{2} \bar{c}_{1}\right)^{2}, \quad A_{34}=\left(c_{3} \overline{\boldsymbol{e}}_{4}-c_{4} \bar{c}_{3}\right)^{2}
$$

D'une manière analogue on obtient pour $* V_{m}^{4}(2,2)$

$$
* K(\pi)=\frac{2}{\lambda^{2}}\left(A_{12}+A_{34}\right)-\frac{1}{\lambda^{2}}
$$


et de là il vient

$$
\frac{K(\pi)}{* K(\pi)}=\lambda^{4},
$$

formule qui donne une interprétation de l'invariant $\lambda$.

Si on suppose que $\boldsymbol{C}$ et $\overline{\boldsymbol{C}}$ sont respectivement le champ unitaire correspondant au champ associé à la 1-forme distinguée $\omega=\sum_{i} \omega^{i}$ et le champ unitaire correspondant au champ de courbure, on voit facilement que $K(\pi)=0-\lambda^{2}$.

Ainsi:

La courbure de sectionnement du champ associé à la 1-forme distinguée et du champ de courbure pour une variété $V_{m}^{4}(2,2)$ est toujours $-\lambda^{2}$.

\section{BIBLIOGRAPHIE}

[1] M. A. AkIvis, The strueture of n-dimensional surfaces carrying a net of ourvature lines, Dokl. Akad. Nauk SSSR, Moseou, 139, pp. 1279-1282.

[2] B. Y. CHEN, On the total curvature of manifolds immersed in Riemannian manifolds, Kodai Mathem. Sem. Rep., 22 (1970).

[3] Cl. Godbtllon, Géométrie différentielle et mécanique analytique, Herman, Paris, 1969.

[4] K. Kenмotsu, Some remarks on minimal submanifolds, Tôhoku Math. Journ., 22 (1970).

[5] A. LichnÉRowicz, Les relations intégrales d'invariance et leurs applications à la dynamique, Bull. Sc. Math., 70 (1946).

[6] R. Osserman, A Survey of Minimal Surfaces, Van Nostrand Reinhold Company, New York, 1969; Bull. Amer. Math. Soo., 75 (1969).

[7] T. OTsukI, Minimal hypersurfaces in a Riemannian manifold of constant eurvature, Amer. Journ. Math., 92 (1970).

[8] T. OTSUKI, On the total curvature of surfaces in Euclidean spaces, Japan J. Math., 35 (1966).

[9] T. OTsUKI, On parallel hypersurfaces of an elliptic hypersurface of the second order in $E^{n+1}$, Tôhoku Mathem. Journ., 19 (1967).

[10] G. REEB, Sur certaines propriétés topologiques des trajectoires des systèmes dynamiques, Bruxelles, Mèmoires Acad. Sc., 27 (1952).

[11] R. Rosca, Sur les hypersurfaces isotropes de défaut 1 incluses dans une variété lorentzienne, C.R. Acad. Se. Paris, série A, 272 (1971), pp. 393-396.

[12] R. RoscA, Sur les espaces-temps cylindriques symmétriques, Rend. Sem. Matem. Messina (sous presse).

[13] R. Rosca, Sur les variétés minimales $V^{2}$ de E. Oartan dans un espaces elliptique à $n$ dimen. sions, Ann. di Mat. pura e appl., serie IV, 87 (1970).

[14] R. RosCA - F. BoREL, Sur les autotransformations infinitésimales équivalentes des variétés minimales à deux dimensions d'un espace elliptique n-dimensionnel, C.R. Acad. Sc. Paris, série A, 268 (1969), pp. 399-401.

[15] R. Rosca, Les hypersurfaces pseudo-isotropes dans un espace de Minkows7i, Bull. Cl. Se. Acad. Roy. Belg., 4 (1970). 
[16] R. RoscA, Les variétés pseudo-isotropes dans un espace-temps de Minkowski, C.R. Acad. Sc. Paris, série A, 270 (1970), pp. 1071-1073.

[17] A. Svec, Projective differential geometry of line congruences, Prague, 1965.

[18] R. TAKaGI, Homogeneous hypersurfaces in a sphere with the type number 2, Tôhoku Math. Journ., 23 (1971).

[19] P. VINCENSINI, Sur le problème des transformations équivalentes infinitésimales d'une surface et ses relations avec la théorie des congruences de sphères, Ann. Scient. Ec. Norm. Sup., $2 \Theta$ série, 79 (1962).

[20] W. O. VoGEL, Veber lineare Zusammenhange in singularen Riemannschen Raumen, Arch. Math., 16 (1965).

[21] E. Bompran, Interno alle varietà isotrope, Ann. di Mat. pura e appl., IV, 20 (1940).

[22] R. Rosca, Les hypersurfaces pseudo-isotropes dans un espace de Minkowsti, Bull. Cl. Sc. Acad. Roy. Belg., 56, no. 4 (1970). 